\title{
AUTOMATED PHOTOGRAMMETRIC METHOD TO IDENTIFY INDIVIDUAL PAINTED DOGS (LYCAON PICTUS)
}

\author{
Kanako Ake*a,b, Tadatoshi Ogura ${ }^{a}$, Yayoi Kaneko ${ }^{c}$ and Gregory S. A. Rasmussen ${ }^{\text {d,e }}$
}

${ }^{a}$ Laboratory of Animal Behavior, Division of Animal Science, School of Veterinary Medicine, Kitasato University, Higashi 23-35-1, Towada, Aomori 034-8628, Japan; ${ }^{b}$ Carnivore Ecology and Conservation Research Group, Graduate School of Agriculture, Tokyo University of Agriculture and Technology, Saiwaicho 3-5-8, Fuchu, Tokyo 183-8509, Japan; ${ }^{\circ}$ Carnivore Ecology and Conservation Research Group, Institute of Agriculture, Tokyo University of Agriculture and Technology, Saiwaicho 3-5-8, Fuchu, Tokyo 183-8509, Japan; dPainted Dog Research Trust, P.O.Box 285, Victoria Falls, Zimbabwe; 'Department of Biological Sciences, University of Zimbabwe, P O Box MP167, Mt Pleasant, Harare, Zimbabwe. *Corresponding author. Email: kanako.ake@gmail.com

\section{Article history Received: 18 March 2019; accepted 1 July 2019}

\section{Keywords:}

African Painted Dog; photo-identification; Hotspotter; Camera trap; non-invasive

\begin{abstract}
The painted dog, Lycaon pictus, has been visually identified by their tricolor patterns in surveys and whilst computerised recognition methods have been used in other species, they have not been used in painted dogs. This study compares results achieved from Hotspotter software against human recognition. Fifteen individual painted dogs in Yokohama Zoo, Japan were photographed using camera-traps and hand-held cameras from October 17-20, 2017. Twenty examinees identified 297 photos visually, and the same images were identified using Hotspotter. In the visual identification, mean accuracy rate was $61.20 \%$, and a mean finish time was 4,840 seconds. At $90.57 \%$, the accuracy rate for Hotspotter was significantly higher, with a mean finish time of 3,168 seconds. This highlights that visual photo-recognition may not be of value for untrained eyes, while software recognition can be useful for this species. For visual identification there was a significant difference in accuracy rates between hand-held cameras and camera-traps whereas for software identification there was no significant difference. This result shows that the accuracy of software identification may be unaffected by the type of photographic device. With software identification there was a significant difference with camera-trap height. This may be because the images of one camera-trap at a lower position became dark due to it being in a shadow.
\end{abstract}

\section{INTRODUCTION}

In the $18^{\text {th }}$ century the painted dog occupied the whole of sub-Saharan Africa, but their numbers have been reduced to $7 \%$ of their original abundance (Marsden et al. 2012) and consequently are now classified as endangered (Woodroffe and Sillero-Zubiri 2012). Conservation-based research requires an understanding of population dynamics and ecology, and the ability to identify individuals is paramount. Consequently, in studying painted dogs researchers have used the species-specific color patterns as a valuable identification tool (Malcolm and Marten 1982; Jordan et al. 2013; Jordan et al. 2014; Jackson et al. 2017), however this is a time-consuming procedure.

Individual recognition using computer software has been achieved for a number of species (whale sharks Megachasma pelagios, Arzoumanian, Holmberg, and Norman 2005; harbor seals Phoca vitulina, Hastings, Hiby, and Small 2008; African penguins Spheniscus demersus, Sherley et al. 2010; giraffes Giraffa camelopardalis, Bolger et al. 2012; Halloran, Murdoch, and Becker 2014; zebras Equus spp., Lahiri et al. 2011; tigers Panthera tigris, Hiby et al. 2009; cheetahs Acinonyx ju- batus, Kelly 2001; slater's skinks Liopholis slateri, Claire et al. 2017; wyoming toads anaxyrus baxteri, Gamble, Ravela, and McGarigal 2008; marbled salamanders Ambystoma opacum, González-Ramos et al. 2017), but none has yet been conducted to identify painted dogs. This study aims to establish a way to identify and process digital images. One such software is Hotspotter, which has been used for zebras, giraffes, jaguars Panthera onca and lionfish Pterois spp. (Crall et al. 2013). In this paper, we conducted a trial to test the relative effectiveness of Hotspotter software for identifying individual painted dogs.

\section{MATERIALS AND METHODS}

\section{Photographic image data}

Fifteen individuals (10 adults 5 yearlings) (Figure 1) were photographed from within and outside their enclosure $\left( \pm 700 \mathrm{~m}^{2}\right)$ at Yokohama Zoo (Zoorasia), Japan from October 17-20, 2017. Two hand-held cameras, NIKON D7500 and CASIO EXLIM EX-ZR1600, and nine camera traps, Ltl-6310M HD Video Series $(\mathrm{n}=8)$, and 
one Fotopasca KeepGuard KG-690NV, were used. The Fotopasca KeepGuard KG-690NV and six Ltl-6310M HD Video Series camera traps photographed the dogs from a higher point above the ground $($ mean $=2.33 \mathrm{~m})$, and two were set lower $($ mean $=1.02 \mathrm{~m})$ (Figure 2).

Camera trap settings were as follows: 12MP (Ltl-6310M HD Video Series), 8MP (Fotopasca KeepGuard KG$690 \mathrm{NV}$ ), three pictures per trigger, 30 second interval after triggering. Only images that included the entire body were used, and equal samples of left and right sides were chosen. This research was conducted after the approval of the animal testing committee and the president of Kitasato University (approval number: 17-174).

\section{Individual recognition using software}

In accordance with the requirements of the software, images used for individual recognition were stored in the Hotspotter software database and rectangularly cropped (Figure 3, Department of Computer Science Rensselaer Polytechnic Institute, www.cs.rpi.edu/hotspotter/). The software identified the images based on descriptors of their body color patterns and uses two algorithms: a) for every image, the algorithms locate key points and extract associated descriptors (128-dimensional vectors), and b) they then determine image matches based on the comparison of these descriptors (Crall et al. 2013). Consequently, ranking was done in order of the similarity scores based on Local Naïve Bayes Nearest Neighbor methods (McCann and Lowe 2012) of descriptors from other images in the database. When the individual of the image identified was the first to be ranked, it was considered as correct. Accuracy rate \% was defined as the number of correct images divided by the total number of images used for the Hotspotter analysis.

\section{Visual recognition}

Twenty students $(6 \hat{\partial}, 14+$ ) aged 20-24 year of age from Kitasato University who had no prior knowledge of the dogs at Yokohama Zoo, were given A4 size printed images and asked to sort and group the individual painted dogs based on their coat patterns. To determine if prior knowl- edge would assist recognition, as the keepers at Yokohama Zoo use the tails to identify the dogs, eight students $(3 \hat{\jmath}, 5$ P) were given this hint. The examinees were divided into two additional groups: one identified the right sides and the other group the left side. In total, we had four groups (Left-Hint, Right-Hint, Left-No hint, Right-No Hint).

Each examinee was given the same 149 or 148 images and there was no time limit to accomplish the recogni-

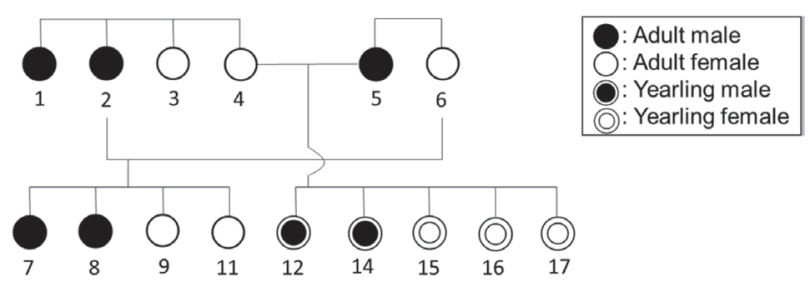

Figure 1. Mates and siblings relations of the painted dogs in Yokohama Zoo (October 2017). The number shows the individual ID (Table 1).

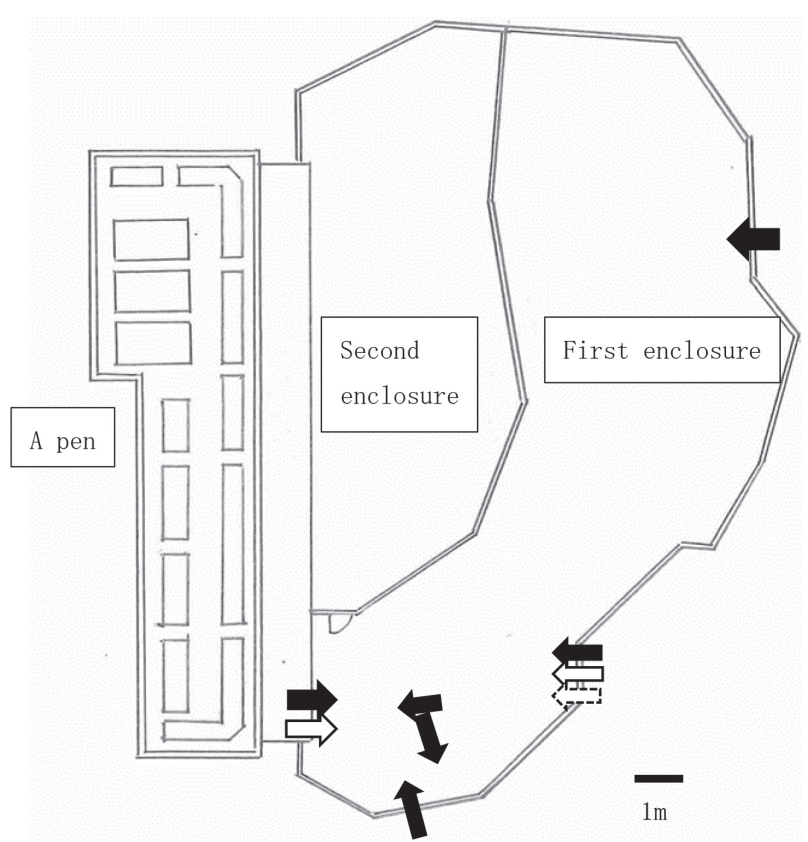

Figure 2. The enclosures and pen.

Note. $\Rightarrow$ : new camera-traps photographed from above; black $\Rightarrow$ : new camera-traps photographed from side; $\Rightarrow$ of dotted line: old camera-traps photographed from above.

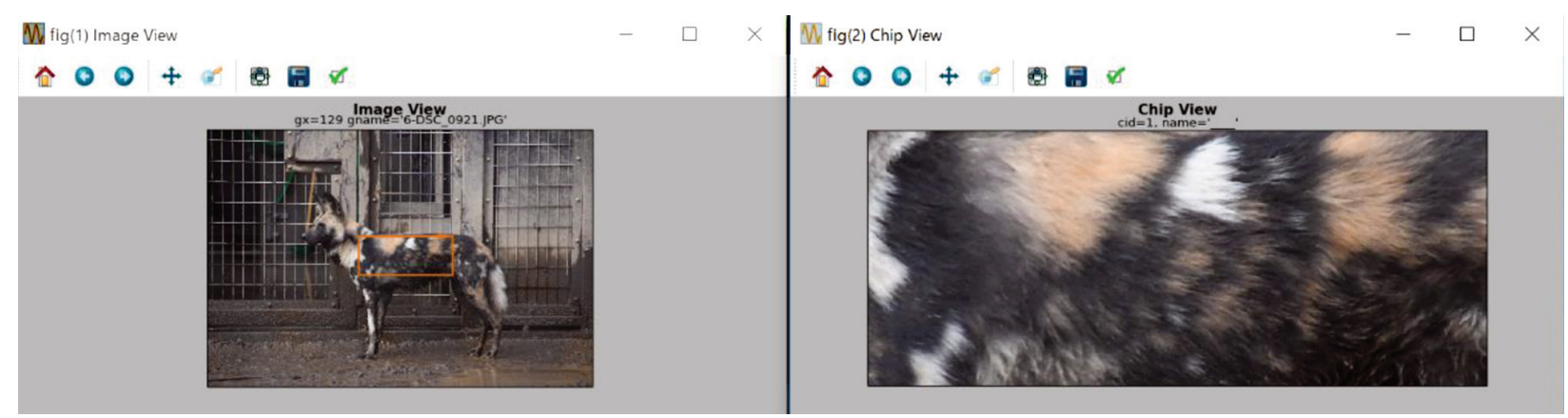

Figure 3. An example of the image used in the software (dog ID is No. 6, taken by the Hand-held camera). Orange rectangular in left picture was the range used for the software recognition from the original photograph. Right picture shows actual range we cropped. 
tion task. The time each student took to complete their task was recorded, and to standardize the timing, those students that had one more image had their times reduced by $0.68 \%$.

We defined the accuracy rate (in percentage) as the number of correctly identified images/divided by the total number of images. Scoring for correct identification was based on:

1. When same individual images were separated into 2 groups and the images were not the same number: -1 point deduction per image of smaller group of the 2 groups

2. When same individual images were separated into 2 groups and the images were the same number: -0.5 point deduction of each image of the 2 groups

3. When same individual images were separated into 3 groups: -1 point deduction of all the images of the 3 groups

4. When the same number image of 2 individual were grouped as same group: -0.5 point deduction of each the images (however, -1 point deduction of each the images when the case fell under No. 1 or 2)

5. When more than 3 individual images were grouped as same group: -1 point deduction of all the images.

\section{Statistical analysis}

Statistical analyses (Wilcoxon signed-rank/rank sum test) were conducted with the software R ver. 2.14.2 (R Development Core Team 2012).

\section{RESULTS}

We captured 22,507 images with the camera traps and 5,840 images with the handheld cameras. A total of 297 images ( 149 of the right sides and 148 of the left sides of the dogs) were suitable for identification (Table 1).

Recognition using Hotspotter had a mean accuracy rate of $90.57 \%$ (269/297 images) and the finish time was 3,168 seconds. There was no significant difference between mean accuracy rate of camera trap photographs (90.54\%, 134/148 images) and hand-held camera images (90.60\%, 135/149 images) $\left(\chi^{2}=0.032, p=0.86\right.$, Figure 4$)$. There also was no significant difference between Ltl camera traps $(91.20 \%, 114 / 125$ images) and Fotopasca camera traps $\left(86.96 \%, 20 / 23\right.$ images, $\chi^{2}=0.016$, $p=0.90$ ), therefore the data acquired from the traps were pooled. However, there was a significant difference $\left(\chi^{2}=6.57, p=0.01\right)$ between the photographs taken from a higher point above the ground ( mean $=95.74 \%, 90 / 94$ images) and those taken from the lower point (mean = $81.48 \%, 44 / 54$ images, Figure 5).

A comparison of the hint group with the no hint group, showed that there was no significant difference in either visual recognition accuracy rate or time to finish (the Wilcoxon rank sum test, $\mathrm{W}=48, p=1.00$, no hint group was $62.01 \%$, hint group was $59.99 \%$ ), and mean finish time (the Wilcoxon rank sum test, $\mathrm{W}=42, p=0.68$, no hint group was 4,939 seconds, hint group was 4,652 seconds). These data could therefore be pooled for the purpose of further statistical analysis.

In visual recognition, the mean accuracy rate was

Table 1. Details of quantities of photograph images used for individual recognition to compare explanatory variables.

\begin{tabular}{|c|c|c|c|c|c|c|c|c|c|c|c|}
\hline \multirow{2}{*}{ No. } & \multicolumn{5}{|c|}{ right side } & \multicolumn{5}{|c|}{ left side } & \multirow{2}{*}{ Total } \\
\hline & above new & side new & above old & hand-held & sub total & above new & side new & above old & hand-held & sub total & \\
\hline No. 1 & 1 & 1 & 1 & 8 & 8 & 1 & 1 & 1 & 7 & 7 & 15 \\
\hline No. 2 & 1 & 1 & 1 & 6 & 6 & 1 & 1 & 1 & 5 & 5 & 11 \\
\hline No. 3 & 4 & 2 & 1 & 4 & 11 & 4 & 1 & 0 & 4 & 9 & 20 \\
\hline No. 4 & 5 & 4 & 3 & 6 & 18 & 5 & 4 & 3 & 4 & 16 & 34 \\
\hline No. 5 & 4 & 0 & 0 & 5 & 9 & 2 & 2 & 1 & 3 & 8 & 17 \\
\hline No. 6 & 4 & 4 & 1 & 5 & 14 & 4 & 5 & 0 & 5 & 14 & 28 \\
\hline No. 7 & 1 & 1 & 1 & 3 & 3 & 1 & 1 & 1 & 5 & 5 & 8 \\
\hline No. 8 & 1 & 1 & 1 & 6 & 6 & 1 & 1 & 1 & 4 & 4 & 10 \\
\hline No. 9 & 1 & 1 & 1 & 4 & 4 & 1 & 1 & 1 & 5 & 5 & 9 \\
\hline No. 11 & 1 & 1 & 1 & 6 & 6 & 1 & 1 & 1 & 4 & 4 & 10 \\
\hline No. 12 & 2 & 1 & 0 & 6 & 9 & 3 & 4 & 0 & 6 & 13 & 22 \\
\hline No. 14 & 2 & 2 & 0 & 5 & 9 & 2 & 2 & 0 & 5 & 9 & 18 \\
\hline No. 15 & 6 & 4 & 2 & 4 & 16 & 6 & 4 & 2 & 6 & 18 & 34 \\
\hline No. 16 & 4 & 4 & 3 & 5 & 16 & 5 & 4 & 3 & 5 & 17 & 33 \\
\hline No. 17 & 4 & 4 & 2 & 4 & 14 & 5 & 3 & 1 & 5 & 14 & 28 \\
\hline Total & 35 & 25 & 12 & 77 & 149 & 36 & 29 & 10 & 73 & 148 & 297 \\
\hline
\end{tabular}

Note: above new: new camera-traps photographed the painted dogs from above; side new: new camera-traps photographed the painted dogs from side; above old: old camera-traps photographed the painted dogs from above; hand-held: hand-held cameras; Diagonal lines indicate we couldn't photograph the painted dogs by camera-traps because of non release in their enclosure. 


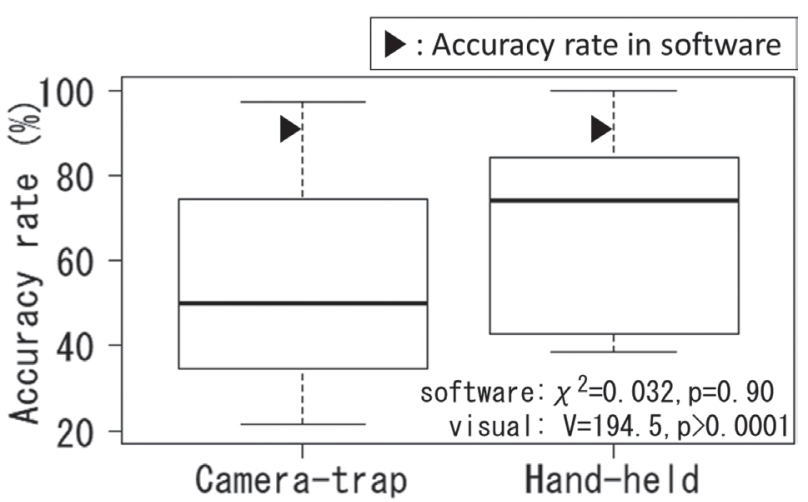

Figure 4. Comparisons of the visual (camera trap and handheld images, boxes) and the software (camera trap and handheld images, black triangles) accuracy rates.

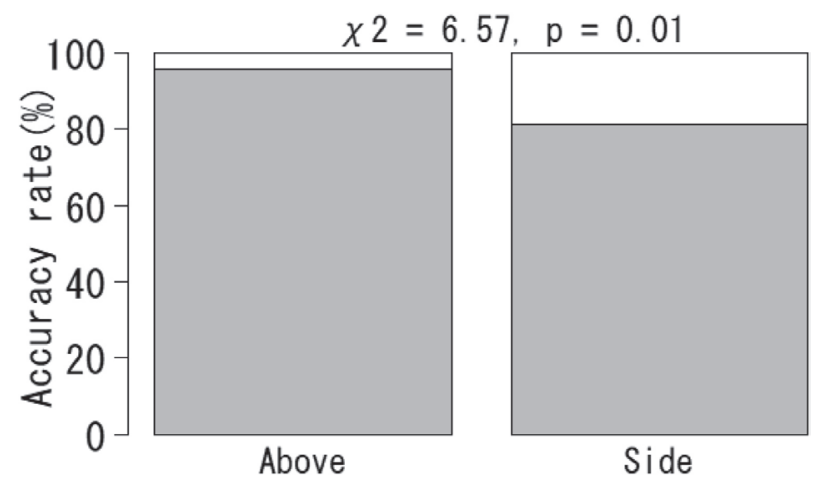

Figure 5. Comparison of accuracy rate (gray, \%) for two camera angles (above, side) in the software recognision.

$61.20 \%$ and the mean finish time was 4,840 seconds. The mean accuracy rate in hand-held cameras was $83.93 \%$ and significantly higher than the $77.25 \%$ rate for camera traps (the Wilcoxon signed-rank sum test, $\mathrm{V}=194.5, \mathrm{p}<$ 0.0001 , Figure 4$)$. There was no significant difference in accuracy rate of the $\mathrm{Ltl}(\mathrm{n}=8,77.54 \%)$ and Fotopasca camera traps $(\mathrm{n}=1,75.65 \%)$ (the Wilcoxon signedrank sum test, $\mathrm{V}=132, \mathrm{p}=0.14)$, therefore the data from these two treatments were pooled. Furthermore, comparison of accuracy rate between the camera trap photographs taken from above (mean $=77.50 \%)$ and from the side $($ mean $=76.81 \%)$ showed no significant difference (the Wilcoxon signed-rank sum test, $\mathrm{V}=127$, $p=0.43)$. The accuracy rate in the software identification was significantly higher than that in visual identification $\left(\chi^{2}=33.43, p<0.01\right)$. Furthermore, the software identification finished 27 minutes and 52 seconds earlier than that by visual identification.

\section{DISCUSSION}

In this study we evaluated the ability of Hotspotter software in individual photo-recognition of painted dogs. The accuracy rate of Hotspotter was high, and as the images photographed by hand-held cameras and the images photographed by camera traps were not significantly different when identified using the software. This indicates that the pixel count didn't affect the result and that both camera types can be used for individual recognition. In the software identification, the accuracy rate from the higher elevation cameras was greater $(95.74 \%)$ and, could be because these images were better than those taken lower down which were more vulnerable to the effect of shadows. Nevertheless, these effects can be mitigated by software, such as photo-edit (i.e. contrast or light edit). In the wild, camera trap images generally are of reasonably high resolution as they are set close to game trails/roads or dens (Rasmussen, G. S. A., unpublished observation, 18 October 2018), and the accuracy rate may well be the same high-quality images from captivity.

We could not detect a significant difference in the accuracy rate and finish time when we compared the hint and no-hint groups in individual recognition. According to our interviews after the examination, almost all of the no-hint examinees answered that they identified the dogs by using the dogs' tail color patterns. It might be possible that they found their own hint. Based on the lower accuracy rate in visual identification, it might be difficult for untrained people to identify the dogs accurately regardless of whether they have a hint. To obtain reliable information using visual identification, training might be necessary.

The accuracy rate of visual identification resulted in $61.20 \%$ copared with $90.57 \%$ for software identification. This may be because the examinees didn't have any prior knowledge about the dogs and had never previously conducted identifications. As accuracy rate will depend on experience, it makes sense that automation and formalization using software can be a very useful and more effective tool. The visual accuracy rate of hand-held camera images was significantly higher than that of camera trap images. This could be because hand-held cameras enabled photographs to be taken which included the entire body color patterns. Moreover, hand-held cameras have a higher pixel count than camera traps and could have photographed the dogs' color patterns more clearly, especially when dogs were far away from camera traps. Hand-held cameras can be useful for recording the dogs' diurnal activity because images of animals may be taken by anyone who has the camera - scientists and their assistants, ecotourists, and even ordinary citizens - increasing the potential for capturing greater quantities of image data (Crall et al. 2013). Camera traps can also be useful, especially for packs of dogs that have not been previously studied, or are located in areas where people cannot easily access. Camera traps also have merit in being able to remotely photograph painted dogs to record their nocturnal behavior when researchers and citizen scientists are 
less active, but the dogs are hunting (Rasmussen and Macdonald 2011).

Comparing the different camera trap photos, we could not detect a significant difference between Ltl-6310M and Fotopasca cameras. At least in our study, we believe the accuracy rate wasn't affected by different camera model types. However, the Ltl-6310M is recommended because it has a wider angle of view and could capture a higher number of images than the Fotopasca.

We did not find a significant difference in the accuracy rates of visual identification between the heights of the camera traps. We consider this is because of the following reasons: Firstly, we chose those images in which we could see their entire bodies to identify them in both high and low conditions and the sunlight didn't affect the result of visual identification. Secondly, a dog's tail wasn't affected by the difference in heights of the camera traps. From this result, we recommend also using visual identification when the software cannot identify the animals because of poor quality images. Kaneko, Koganezawa, and Maruyama (2004) compared the number of species and their frequencies in Okunikkou, Japan by setting camera traps at two elevations (at a height of 4 meter and 0.5 meter). They suggested that placing camera traps at a height of $4 \mathrm{~m}$ is more efficient at verifying more species than at a height of $0.5 \mathrm{~m}$. However, photographing at a height of $4 \mathrm{~m}$ mostly resulted in images of only the back of the target species so we think it's not suitable for painted dogs since they have species-specific color patterns on their legs and flank. We also think that photographing at a height of $0.5 \mathrm{~m}$ should narrow the number of dogs we can photograph at once. For these reasons, we recommend photographing them at a middle height between $0.5-4 \mathrm{~m}$.

In this captive study we established how to use software to identify painted dogs by their species-specific color patterns, however, it would be good to test this against a "wild" dataset as ultimately the ability to accurately identify individuals in the wild is what will facilitate their conservation.

\section{CONCLUSION}

Our data suggest that with good photographs, Hotspotter can be useful as a rapid assessment tool to facilitate recognition. Furthermore, in the wild, camera traps generally are set in such a way as to get close images (e.g., at dens or game trails) and it is highly probable these images will be as accurate as those taken by hand held cameras. To find the best tool to identify the dogs, the following future studies are recommended:

1. test camera trap images taken by researchers undertaking wild studies
2. test different cropping of the images and alternative software to find out if higher mean score than $90.57 \%$ can be achieved.

\section{ACKNOWLEDGEMENTS}

We would like to thank Ms. M. Ishida and Mr. E. Kawaguchi, Yokohama Zoo and the 20 examinees in Kitasato University agreed for assistance and research on this study. Thanks also to Dr. A. Matsuura and Ms. A. Hirata for valuable comments. We are grateful to Ms. T. Cloutier for assistance with English expression. Finally, the authors would like to thank the referees for useful comments.

\section{REFERENCES}

Arzoumanian, Z., J. Holmberg, and M. Norman. 2005. 'An astronomical pattern-matching algorithm for computer-aided identification of whale sharks Rhincodon typus. 'Journal of Applied Ecology 42: 999-1011.

Bolger, D. T., T. A. Morrison, B. Vance, D. Lee, and H. Farid. 2012. 'A computer-assisted system for photographic mark-recapture analysis.' Methods in Ecology and Evolution 3: 813-822.

Claire, E. T., R. P. Chris, N. H. Mark, and M. C. Bull. 2017. 'Photographic recognition of individuals of a free-ranging, small terrestrial vertebrate.' Ecology and Evolution 6: 800-809.

Computer Science Science at Rensselaer, Department of Computer Science Rensselaer Polytechnic Institute. 2013. "Hotspotter." Accessed July 132018.

Crall, J. P., C. V. Stewart, T. Y. Berger-Wolf, D. I. Rubenstein, and S. R. Sundaresan. 2013. Hotspotter: Patterned Species Instance Recognition. Institute of Electrical and Electronic Engineers Workshop on Applications of Computer Vision.

Gamble, L., S. Ravela, and K. McGarigal. 2008. 'Multiscale features for identifying individuals in large biological databases: An application of pattern recognition technology to the marbled salamander Ambystoma opacum.' Journal of Applied Ecology 45: 170-180.

González-Ramos, M. S., A. Santos-Moreno, E. F. RosasAlquicir, and G. Fuentes-Mascorro. 2017. 'Validation of photo-recognition as a mark-recapture method in the spotted eagle ray Aetobatus narinari.' Fish Biology 90: 1021-1030.

Halloran, K. M., J. D. Murdoch, and M. S. Becker. 2014. 'Applying computer-aided photo-identification to messy datasets: A case study of Thornicroft's giraffe Giraffa camelopardalis thornicrofti.' African Journal of Ecology 53: 147-155. 
Hastings, K. K., L. A. Hiby, and R. J. Small. 2008. 'Evaluation of a computer-assisted photograph-matching system to monitor naturally marked harbor seals at Tugidak Island, Alaska.' Journal of Mammalogy 89: 1201-1211.

Hiby, L., P. Lovell, N. Patil, N. S. Kumar, A. M. Gopalaswamy, and K. U. Karanth. 2009. 'A tiger cannot change its stripes: using a three-dimensional model to match images of living tigers and tiger skins.' Biology Letters 5 (3): 383-386.

Jackson, C. R., R. J. Groom, N. R. Jordan, and J. W. McNutt. 2017. 'The effect of relatedness and pack size on territory overlap in painted dogs.' Movement Ecology 5 (10) (DOI: 10.1186/s40462-017-0099-8, 2019/07/06).

Jordan, N. R., P. J. Apps, K. A. Golabek, and J. W. McNutt. 2014. 'Top marks from top dogs: Tandem marking and pair bond advertisement in painted dogs.' Animal Behaviour 88: 211-217.

Jordan, N. R., K. A. Golabek, P. J. Apps, G. D. Gilfillan, and J. W. Mcnutt. 2013. 'Scent-mark recognition and scent-marking behaviour in painted dogs (Lycaon pictus).' Ethology 119: 644-652.

Kaneko, K., M. Koganezawa, and T. Maruyama. 2004. 'Difference between automatic photographing two methods and spotlight census method in species and the number observed.' Wildlife Research Report 30: 34-42 (In Japanese).

Kelly, M. J. 2001. 'Computer-aided photograph matching in studies using individual identification: An example from Serengeti cheetahs.' Journal of Mammalogy 82: 440-449.

Lahiri, M., C. Tantipathananandh, R. Warungu, D. I. Rubenstein, and T. Y. Berger-Wolf. 2011. 'Biometric animal databases from field photographs: Identification of individual zebra in the wild.' Proceedings of the 1st Association for Computing Machinery International Conference on Multimedia Retrieval.

Malcolm, J. R. and K. Marten. 1982. 'Natural selection and the communal rearing of pups in painted dogs (Lycaon pictus).' Behavioral Ecology and Sociobiology 10: 1-13.

Marsden, C. D., R. Woodroffe, M. G. L. Mills, J. W. McNutt, S. Creel, R. Groom, M. Emmanuel, S. Cleaveland, P. Kat, G. S. A. Rasmussen, J. Ginsberg, R. Lines, J. M. Andre, C. Begg, R. K. Wayne, and B. K. Mable. 2012. 'Spatial and temporal patterns of neutral and adaptive genetic variation in the endangered painted dog (Lycaon pictus).' Molecular Ecology 21: 1379-1393.

McCann, S., and D. G. Lowe. 2012. 'Local naïve bayes nearest neighbor for image classification.' Institute of Electrical and Electronic Engineers Computer Society Conference on Computer Vision and Pattern Recognition (DOI: 10.1109/CVPR.2012.6248111, 2019/07/06).

R Development Core Team. 2012. R: A language and environment for statistical computing. Vienna: R Foundation for Statistical Computing.

Rasmussen, G. S. A., and D. W. Macdonald. 2011. 'Masking of the zeitgeber: Painted dogs mitigate persecution by balancing time.' Journal of Zoology 286: 232-342.

Sherley, R. B., T. Burghardt, P. J. Barham, N. Campbell, and I. C. Cuthill. 2010. 'Spotting the difference: Towards fully-automated population monitoring of African penguins Spheniscus demersus.' Endangered Species Research 11: 101-111.

Woodroffe, R. and C. Sillero-Zubiri. 2012. Lycaon pictus. The IUCN Red List of Threatened Species 2012. 